\title{
Comparison of in vitro pharmacodynamics equivalence of generic and branded antimicrobials
}

\begin{abstract}
Akemfua Fualefac ${ }^{1}$, Michel Kengne ${ }^{1}$, Valentine Ndikum ${ }^{2,3}$, Achiangia Patrick Njukeng ${ }^{4}$, Ubald Tamoufe $^{5}$, Daniel Ter Goon ${ }^{6}$, Cedric Mbakam Happi ${ }^{1}$, Joel Ngoupa ${ }^{1}$, Awung Nkeza ${ }^{1}$, Marcellin Ndoe Guiaro ${ }^{7}$ and Julius Mbekem Nwobegahay ${ }^{1,8 *}$
\end{abstract}

${ }^{1}$ Department of Microbiology/Immunology, School of Health Sciences, Catholic University of Central Africa, Yaounde, Cameroon.

${ }^{2}$ Faculty of Medicine and Biomedical Sciences, University of Yaoundé I, Cameroon.

${ }^{3}$ Biotechnology Centre, University of Yaoundé I, Cameroon.

${ }^{4}$ Department of Microbiology, University of Buea, Buea, Cameroon.

${ }^{5}$ Metabiota Cameroon, Yaounde, Cameroon.

${ }^{6}$ Department of Nursing Sciences, University of Fort Hare, South Africa.

${ }^{7}$ Institute of Medical Research and Studies of Medicinal Plants, Ministry of Scientific Research and Innovation, Yaounde, Cameroon.

${ }^{8}$ Military Health Research Center (CRESAR), Yaounde, Cameroon.

Accepted 5 October, 2018

\begin{abstract}
There has been an enormous increase in the clinical use of generic drugs. However, this has not been accompanied by frequent and systematic evaluations of their therapeutic efficacy compared with branded products. Although essential to reduce health budget costs and to promote pharmaceutical competition, generic drugs remain a topic of intense controversy. Generics are usually intended to be inter-changeable with branded products; however, there have been allegations that some have a lower therapeutic equivalence than their branded counterparts The main objective of this study was to compare the in vitro potency of generic and branded antibiotics on E. coli and S. aureus. A sample of generic and corresponding branded Ciprofloxacin, Ceftriazone, Gentamicin and Doxycycline were used to prepare antibiotic disk for Kirby-Bauer disk diffusion susceptibility antimicrobial assay on E. coli ATCC 25922 and S. aureus ATCC 25923. The inhibition zones were measured and relative potencies were calculated. Antimicrobial activity against S. aureus ATCC 25923 ranged from 92.50 to $100 \%$ for generic agents; and $96.89 \%$ to $100 \%$ for branded drugs. The potency range for E. coli ATCC 25922 were 86.61 to $104.35 \%$ for generics and 83.33 to $103.23 \%$ for branded antimicrobials. There was no significant difference between the potency of the generic and the branded antimicrobial agents on the bacterial strains in the study. Each generic antimicrobial agent presented relative potency that was within the $80-125 \%$ acceptable range. The in vitro pharmacodynamics potency of generic antimicrobials from health facilities and legal distributors are equivalent to their corresponding branded agents.
\end{abstract}

Keywords: Antibiotics, antimicrobial activity, potency equivalence, generic antibiotics, branded antibiotics.

${ }^{*}$ Corresponding author. E-mail: nwobegahay@yahoo.com.

\section{INTRODUCTION}

Important infections caused by bacteria that have become resistant to commonly used antimicrobials have become a major healthcare problem in the $21^{\text {st }}$ century
(Alanis, 2005). Anti-microbial drugs are widely effective for the management and control of various infectious diseases. Such agents have contributed enormously in 
the reduction of the morbidity and mortality associated with infectious diseases. Improving the accessibility to generic drugs will greatly improve treatment outcome in less developed countries where infectious diseases are highly burdened.

There has been an enormous increase in the clinical use of generic antibiotic drugs over the years. Between 2000 and 2010, consumption of antibiotic drugs increased by $35 \%$ (from 52,057,163,835 standard units to $70,440,786,553$ ) (Van Boeckel et al., 2014). However, frequent and systematic evaluations of the therapeutic efficacy of such agents are not conducted periodically as needed compared with branded products. Although it is the major aim to reduce health budget costs and to promote pharmaceutical competition and employment, generic drugs remain a topic of intense controversy as a result of the accelerated approval process for human use, and sporadic reports of failures and deaths associated with their use.

In order to improve the accessibility of the population to antimicrobial therapy, generic antimicrobials are being produced and distributed at lower costs than the original innovative branded products. A generic is defined as a drug product that is comparable to a brand drug product in dosage form, strength, route of administration, quality and performance characteristics, and intended use (Carillo and Postigo, 2012). Generic antimicrobials are widely prescribed in less developed countries for the treatment of mild to severe infections. Most of the drugs do not undergo quality assurance process and the manufacturers are not regulated. Even though generics are usually intended to be inter-changeable with the branded products, there have been allegations that some have a lower therapeutic equivalence than their branded counterparts (Kiron et al., 2011). Patients on branded anti-epileptic drugs were observed to have recurrence of their seizures after their drug regimen was switched to generics. In addition, there are recently published arguments suggesting that generic antibiotics do not present the full reliability needed to claim therapeutic equivalence with branded drugs (Gauzit and Lakdhari, 2012).

There are differences at all levels between branded and generic drugs. Some of these include: drug components, level of purity, pharmacokinetics, pharmacokinetics/pharmacodynamics relationship, in vitro effectiveness, therapeutic effectiveness in experimental models, etc (Gauzit and Lakdhari, 2012).

Cameroon does not have an adequate supply of drugs and the introduction of Generic drugs in Cameroon has not been long. Government and church-run hospitals procure some supplies at reasonable prices through international tenders. But the tenders do not allow them to meet all of their needs. Many drugs of low and dubious quality are imported illegally. At the same time, many pharmaceuticals or medical aids such as infusion packs are exported to Cameroon at inflated prices. Cinpharm company manufactures generic drugs in Cameroon and Its first products were distributed to the market in October 2010 (Development and Cooperation, 2010).

Few studies have evaluated the pharmacodynamics or therapeutic equivalence of locally available generic antimicrobial agents in Cameroon. The main aim of this study was therefore to assess the quality and potency of some generic antibiotics compared to the corresponding branded forms used in Cameroon. The results obtained will assist in the fight against antibiotic drug resistance because the consumption of poor quality generics will lead to an enormous increase in the rate of antibiotic drug resistance within the community.

We therefore used an in vitro antimicrobial assay to compare pharmacodynamics equivalence of some generic and branded antimicrobial agents obtained in Yaounde. The efficacies of generic antimicrobials have to be ascertained as they are widely used for the treatment of infectious diseases. This could be very important in improving the confidence in generic antimicrobials. As such, available generics will be widely prescribed and infectious diseases could be better managed.

\section{MATERIAL AND METHODS}

\section{Study design}

A sample of 11 generic antibiotic tablets (Ciprofloxacin - 2, Ceftriazone - 2, Gentamicin - 4 and Doxycycline - 3) from different manufacturers was purchased from four different retail pharmacies and a Health Center in Yaounde. Their corresponding branded drugs were obtained from a pharmacy. Information was collected on batch number, date of production and date of expiration. Antibiotic disks with different concentrations $(5,10,15,20,25$ and $30 \mu \mathrm{g}$ ) were produced using these antibiotics based on methods already described by Epoke et al. (2003).

\section{Calculation of relative potency}

Antibiotic disks of $30 \mu \mathrm{g}$ of each drug and the control disks which also had $30 \mu \mathrm{g}$ of antibiotic were used to calculate the percentage loss in potency, from which the potency of the drug is obtained as shown in the following equation:

$$
\text { Percentage loss in potency }=\frac{(\text { diameter standard drug }- \text { diameter generic drug })}{\text { Diameter of standard drug }} \times 100
$$

Potency of each antibiotic $=100-$ percentage loss in potency of each antibiotic

(Zuluaga et al., 2009). Following incubation, the zone size in millimeter was measured using a graduated scale. The diameter of the disk was considered as a standard in the measurement. The zone of clearance was rounded up to the next millimeter. 


\section{Antimicrobial assay}

E. coli (ATCC 25922) and S. aureus (ATCC 25923) species were used for the antimicrobial susceptibility bioassay. Kirby-Bauer disk diffusion susceptibility method was used and culture media (Mueller Hinton agar and broth, Nutrient agar) was prepared based on the manufacturer's manual (Zuluaga et al., 2009). The strains (control strains of $S$. aureus and $E$. coli) were grown on nutrient agar plates for $24 \mathrm{~h}$ at $37^{\circ} \mathrm{C}$ and further sub cultured in Mueller Hinton broth for $24 \mathrm{~h}$ at $37^{\circ} \mathrm{C}$, then inoculated on Mueller Hinton agar and incubated for $24 \mathrm{~h}$ at $37^{\circ} \mathrm{C}$ in order to have discrete colonies.

The inoculum was prepared by using a sterile inoculating loop to touch 4 or 5 isolated colonies of the organism to be tested, and resuspending the organisms in 3 to $5 \mathrm{ml}$ of sterile saline (Crosse et al., 1981). The suspension was then vortexed to create a smooth suspension and the turbidity of the suspension was adjusted to a $0.5 \mathrm{McF}$ arland standard $\left(\approx 10^{8} \mathrm{CFU} / \mathrm{ml}\right.$ ) by adding more organisms if too light, or by diluting with sterile saline if the suspension was too heavy. This suspension was used within 15 min after preparation (Hudzicki, 2012; Sekowska et al., 2011).

Inoculation of Mueller Hinton plates was done by dipping a sterile swab in the inoculum tube and rotating the swab against the side of the tube (above the fluid level) using firm pressure, to remove excess fluid. Care was taken to prevent it from dripping wet. The dried surface of the agar plate was inoculated by streaking the swab three times over the entire agar surface and rotating the plate approximately 60 degrees each time to ensure an even distribution of the inoculum. The plate was rimed with the swab to pick up any excess liquid. The swab was then discarded into an appropriate container and the plate kept slightly opened at room temperature at least for 3 to $5 \mathrm{~min}$ (but not more than $15 \mathrm{~min}$ ) for the surface of the agar to dry before proceeding to the next step (Hudzicki, 2012).

The appropriate antimicrobial-impregnated disks were placed on the surface of the agar using forceps to dispense each disk, one at a time (Figure 1). Disks were not placed close to the edge of the plate and were not closer than $24 \mathrm{~mm}$. Each disk was pressed down with the forceps to ensure direct contact with the agar surface in order to prevent the formation of irregular zone shapes. Seven antibiotic disks were placed in each plate including one control disk; that is, $5,10,15,20,25$ and $30 \mu \mathrm{g}$ disk with a control disk of $30 \mu \mathrm{g}$ of the same drug on each plate. The assay was done twice for each drug on the different microorganism using separately prepared inocula (Hudzicki, 2012).

\section{Statistical analysis}

Data were analyzed using the Statistical package for the social sciences (SPSS version 17.0; Chicago, USA). Significant differences between categorical variables were determined by analysis of variance (ANOVA). A p value of $<0.05$ was considered statistically significant.

\section{RESULTS}

A total of 11 generics and 4 branded antibiotics were obtained and successfully tested on control strains of $S$. aureus and $E$. coli. The percentage sensitivity of the quality control organism (S. aureus ATCC 25923) ranged from 92.50 to $100 \%$ for generic agents; and 96.89 to $100 \%$ for branded drugs. Meanwhile, the percentage sensitivity of the quality control organism (E. coli ATCC 25922) ranged from 86.61 to $104.35 \%$ for generics and 83.33 to $103.23 \%$ for branded antimicrobials (Table 1 ).

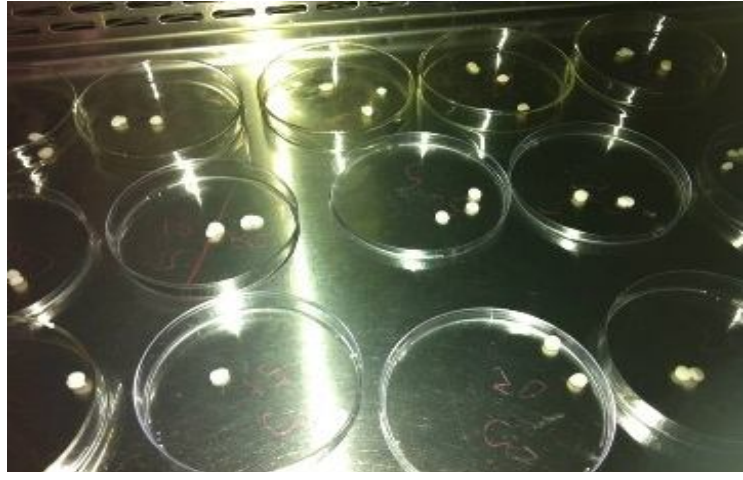

Figure 1. Drying of prepared antibiotic disks in a level 2 biosafety cabinet.

There was no significant difference between the potency of the generic and the branded antimicrobial agents on the bacterial strains used in the study. Each generic antimicrobial agent presented relative potency that was within the 80 to $125 \%$ range, as recommended by the World Health Organization (WHO, 2018).

Statistical analysis revealed that at $95 \%$ confidence interval, the potency of generics ranged from 93.95 to $101.01 \%$; while the branded ranged from 79.42 to $108.69 \%$ on E. coli. Using the same confidence interval, the potency of generics ranged from 95.02 to $98.92 \%$; while that of branded ranged from 96.89 to $95.18 \%$ on $S$. aureus (Table 2). Further evaluation of the potencies of individual antibiotics on both $E$. coli and $S$. aureus revealed that generic ciproflaxin had a potency range of 96.55 to $100.00 \%$ sensitivity on both $E$. coli and $S$. aureus; while branded ciprofloxacin had a potency of 96.88 to $103.23 \%$ sensitivity. Branded formulations of gentamicine and doxycycline presented values of $95 \%$ confidence intervals of 47.40 to $139.14 \%$ and -14.24 to $197.57 \%$, both of which were outside the lower and upper 80 to $125 \%$ sensitivity limits (Table 3 ).

\section{DISCUSSION}

The results of this study show that generic antibiotics have the same in vitro potency as branded antibiotics, and there was no significant difference between the potency of the generic and the branded antimicrobial agents on the bacterial strains in the study. Each generic antimicrobial agent presented relative potency that was within the 80 to $125 \%$ acceptable range (Leelarasamee et al., 2008). The above results were similar to those of Raceme et al. (2013), who had a potency range of 90.00 to $110.00 \%$ for generic cephalosporines (cefixime), and 90.00 to $106.00 \%$ for tetracyclines. The results can further be matched with those of Zuluaga et al. (2009) in which they compared the in vitro anti-microbial parameters of 22 generic products and had a potency estimate of 99.8 to $100.5 \%, 99.7$ to $100.2 \%$ and 98.5 to 
Table 1. Mean relative potencies of all generics and branded antimcrobials with respect to the reference standard on E. coli.

\begin{tabular}{lccc}
\hline Parameter & Generics & Branded & Reference standard \\
\hline Mean potency & 97.48 & 94.06 & $100 \%$ \\
Arithmetic range & $82.61-104.35$ & $83.33-103.23$ & - \\
$95 \%$ Confidence interval & $93.95-101.01$ & $79.42-108.69$ & - \\
\hline
\end{tabular}

Table 2. Mean relative potencies of all generics and branded antimcrobials with respect to the reference standard on S. aureus.

\begin{tabular}{lccc}
\hline Parameter & Generics & Branded & Reference standard \\
\hline Mean potency & 96.97 & 97.67 & $100 \%$ \\
Arithmetic range & $92.5-100$ & $96.89-100$ & - \\
$95 \%$ Confidence interval & $95.02-98.92$ & $95.18-100.14$ & - \\
\hline
\end{tabular}

Table 3. Comparison of mean relative potencies of generic versus specialty agents of antimicrobial agent against both $E$. coli and $S$. aureus.

\begin{tabular}{|c|c|c|c|c|c|c|c|c|}
\hline \multirow[b]{2}{*}{ Parameter } & \multicolumn{2}{|c|}{ Ciprofloxacine } & \multicolumn{2}{|c|}{ Ceftriaxone } & \multicolumn{2}{|c|}{ Gentamicine } & \multicolumn{2}{|c|}{ Doxycycline } \\
\hline & Generic & Branded & Generic & Branded & Generic & Branded & Generic & Branded \\
\hline Mean potency & 97.47 & 100.05 & 98.39 & 99.22 & 97.38 & 98.27 & 96.02 & 91.67 \\
\hline Arithmetic range & $96.55-100.00$ & $96.88-103.23$ & $96.77-100.00$ & $96.88-100.00$ & $92.50-104.17$ & $89.66-96.88$ & $82.61-104.35$ & $83.33-100.00$ \\
\hline 95\% Confidence interval & $94.78-100.15$ & $59.71-140.40$ & $95.42-101.35$ & $96.73-101.70$ & $94.76-99.99$ & $47.40-139.14$ & $88.01-104.03$ & $-14.24-197.57$ \\
\hline
\end{tabular}

$99.9 \%$ for generic products of amikacin, gentamicin and vancomycin, respectively. Also, based on the United States Pharmacopeia (USP), any antibiotic is considered efficient if potency falls within limits of 67 to $150 \%$ (Crosse et al., 1981).

Our results provide evidence for in vitro pharmacodynamics equivalence of the generic antibiotics to their corresponding branded products. This study was limited by the fact that the sample of generic drugs was small compared to that of Zuluaga et al. (2009) and because of administrative constrains (their sources were limited to authorized health centres and retail pharmacies). Other parameters such as the minimum inhibitory concentrations, minimum bactericidal concentration, critical concentrations and the production of spontaneous mutants that are resistant to the antibiotics used in the study were not tested.

According to Le (2014), bioequivalence indicates that the drug products, when given to the same patient in the same dosage, result in equivalent concentrations of drug in plasma and tissues. This bioequivalent study (pharmacokinetics) was not carried out in our study and this will have different results in each individual even if the drugs had equivalent in vitro potencies.

Potent generic drugs will greatly improve healthcare in Cameroon and can be easily afforded by patients with limited income. In addition, the provision of cheap and potent 
generic antibiotics will aid in curbing antibiotic résistance in Cameroon.

\section{Conclusion}

The in vitro antimicrobial activities of generic drugs tested (Ciprofloxacin, Ceftriaxone, Gentamicin and Doxycycline) in this study were equivalent to those of the branded drugs and both forms could be used interchangeably without the fear of the development of antibiotic resistance, as the therapeutic effects are expected to be similar.

\section{REFERENCES}

Alanis AJ, 2005. Resistance to Antibiotics: Are We in the PostAntibiotic Era? Archives of Medical Science, 36: 697-705.

Carillo JA, Postigo MS, 2012. Generic drugs: we must cut pharmaceutical spending but undertaking drug quality. Rev Enferm, 35: 10-9.

Crosse R, Burt DG, Gardner MJ, 1981. Ceftazidime susceptibility testing-disc loading. J Antimicrob Chemother, 8: 217-219.

Development and Cooperation, 2010. Health care: one giant leap. https://www.dandc.eu/en/article/cinpharm-starts-producing-genericdrugs-cameroon (Accessed on October 3, 2018).

Epoke J, Igumbor EO, Asuquo AE, Tichawowona C, 2003. Locally prepared antibiotic sensitivity discs: a substitute for imported commercial discs. Glob J Appl Sci, 9: 453-456.

Gauzit R, Lakdhari M, 2012. Generic drugs: is effectiveness guaranteed? Medecine et maladies infectieuses, 42: 141-148.

Hudzicki J, 2012. Kirby-Bauer Disk Diffusion Susceptibility Test Protocol. Available from: www.microbelibrary.org. (Accessed on January 10, 2018).

Kiron SS, Shirwaikar A, Saritha M, 2011. Influence of storage conditions on the potency of amoxicillin dispersible tablets stored in hospital and community pharmacies in different pharmacies in different regions of Kerala. Asian J Pharmaceut Clin Res, 4: 101-102.

Le J, 2014. Drug Bioavailability - Clinical Pharmacology - MSD Manual. https://www.msdmanuals.com.

Leelarasamee A, Sathirakul K, Trakulsomboom S, Pongpech $P$, Naenna P, 2008. Bioequivilence study with a comparative antibacterial activity of a generic mesopenem. J Infect Dis Antimicrob Agents, 25: 63-72.

Raceme ZM, Siddique AB, Babar SE, 2013. Chemical and microbial analysis of post marketed antibiotics. Greener J Med Sci, 3: 98-110.

Sekowska A, Gospodarek E, Kaminska D, 2011. Antimicrobial susceptibility and genetic similarity of ESBL - positive Klebsiella pneumoniae strains. Arch Med Sci, 6: 993-997.
Van Boeckel TP, Gandra S, Ashok A, Caudron Q, Grenfell BT, Levin SA, Laxminarayan R, 2014. Global antibiotic consumption 2000 to 2010: an analysis of national pharmaceutical sales data. Lancet Infect Dis, 14(8): 742-750.

WHO, 2018. Essential medicines and health products. Norms, guidance and standards for pharmaceuticals. http://www.who.int/medicines /areas/quality_safety/quality_assurance/norms_standards/en/ (Accessed on January 13, 2018).

Zuluaga AF, Agudelo M, Rodriguez CA, Vesga O, 2009. Application of microbiological assay to determine pharmaceutical equivalence of generic intravenous antibiotics. BMC Clin Pharm, 9: 1472-6904.

Citation: Fualefac A, Kengne M, Ndikum V, Njukeng AP, Tamoufe U, Goon DT, Happi CM, Ngoupa J, Nkeza A, Guiaro MN, Nwobegahay JM, 2018. Comparison of in vitro pharmacodynamics equivalence of generic and branded antimicrobials. Microbiol Res Int, 6(4): 49-53. 